\title{
Community-based interventions for the prevention and control of helmintic neglected tropical diseases
}

\author{
Rehana A Salam, Hasina Maredia², Jai K Das', Zohra S Lassi ${ }^{1}$ and Zulfiqar A Bhutta ${ }^{3, *^{*}}$
}

\begin{abstract}
In this paper, we aim to systematically analyze the effectiveness of community-based interventions (CBIs) for the prevention and control of helminthiasis including soil-transmitted helminthiasis (STH) (ascariasis, hookworms, and trichuriasis), lymphatic filariasis, onchocerciasis, dracunculiasis, and schistosomiasis. We systematically reviewed literature published before May 2013 and included 32 studies in this review. Findings from the meta-analysis suggest that CBls are effective in reducing the prevalence of STH (RR: $0.45,95 \%$ Cl: $0.38,0.54$ ), schistosomiasis (RR: $0.40,95 \% \mathrm{Cl}: 0.33,0.50)$, and STH intensity (SMD: $-3.16,95 \mathrm{Cl}$ : $-4.28,-2.04)$. They are also effective in improving mean hemoglobin (SMD: 0.34, 95\% Cl: 0.20, 0.47) and reducing anemia prevalence (RR: 0.90, 95\% Cl: 0.85, 0.96). However, it did not have any impact on ferritin, height, weight, low birth weight (LBW), or stillbirths. School-based delivery significantly reduced STH (RR: $0.49,95 \%$ Cl: $0.39,0.63$ ) and schistosomiasis prevalence (RR: $0.50,95 \%$ Cl: 0.33 , 0.75), STH intensity (SMD: $-0.22,95 \% \mathrm{Cl}:-0.26,-0.17)$, and anemia prevalence (RR: $0.87,95 \% \mathrm{Cl}: 0.81,0.94$ ). It also improved mean hemoglobin (SMD: 0.24, 95\% Cl: 0.16, 0.32). We did not find any conclusive evidence from the quantitative synthesis on the relative effectiveness of integrated and non-integrated delivery strategies due to the limited data available for each subgroup. However, the qualitative synthesis from the included studies supports community-based delivery strategies and suggests that integrated prevention and control measures are more effective in achieving greater coverage compared to the routine vertical delivery, albeit it requires an existing strong healthcare infrastructure. Current evidence suggests that effective community-based strategies exist and deliver a range of preventive, promotive, and therapeutic interventions to combat helminthic neglected tropical diseases (NTDs). However, there is a need to implement and evaluate efficient integrated programs with the existing disease control programs on a larger scale throughout resource-limited regions especially to reach the unreachable.
\end{abstract}

Keyword: Neglected tropical diseases, Soil-transmitted helminthiasis, Community-based interventions

\section{Multilingual abstracts}

Please see Additional file 1 for translations of the abstract into the six official working languages of the United Nations.

\section{Introduction}

Helminths (Greek, meaning 'worms') are parasitic worms that have been harboring in humans throughout the

\footnotetext{
*Correspondence: zulfiqar.bhutta@aku.edu

${ }^{3}$ Center of Excellence in Women \& Child Health, The Aga Khan University, Karachi, Pakistan

${ }^{4}$ Center for Global Child Health Hospital for Sick Children, Toronto, Canada Full list of author information is available at the end of the article
}

ages. These are classified as nematodes or roundworms and include soil-transmitted helminths (ascariasis, hookworms, and trichuriasis) and filarial (causing lymphatic filariasis [LF] and onchocerciasis), whereas the platyhelminthes or flatworms include the flukes (schistosomes) and tapeworms. As discussed in Paper 1 [1], helminthic infections are a prominent subgroup within neglected tropical diseases (NTDs), primarily perpetuated due to a lack of access to safe water and sanitation. The most common helminths are the soil-transmitted helminthiasis (STH), followed by schistosomiasis and LF. These are often co-infectious, although the biology of each disease differs. These infections disproportionately affect children, pregnant women, and young adults resulting in

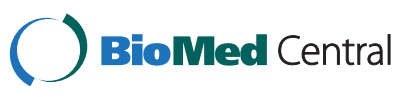


serious chronic health conditions including malnutrition, physical and intellectual growth retardations in children, and adverse maternal, perinatal and delivery outcomes among pregnant women [2-4]. Onchocerciasis is a leading cause of blindness and skin disease, while LF is a major cause of limb and genital deformities. For a more thorough discussion on the epidemiology and burden of each of these diseases, please refer to our previous publication [1].

Mass drug administration (MDA) with anthelminthics has been a major approach to combat human helminthiasis while parallel interventions such as micronutrient supplementation, especially iron, to combat associated anemia has also been widely evaluated for effectiveness [5]. Sanitation and education are also recommended, however, these are not always feasible in resource-limited settings. All these strategies can be effectively administered via community delivery platforms. Child health days, micronutrient supplementation, vaccination programs, and school-based programs provide a potential entry point for periodic deworming and health education in a more cost-effective manner [6]. In this paper, we aim to systematically analyze the effectiveness of community-based interventions (CBIs) for the prevention and control of helminthiasis including STH (ascariasis, hookworms, and trichuriasis), LF, onchocerciasis, dracunculiasis, and schistosomiasis.

\section{Methods}

We systematically reviewed literature published before May 2013 to identify studies on the effectiveness of the community-based delivery of interventions highlighted in our conceptual framework [7] for the outlined helminthic diseases. Our priority was to select existing randomized, quasi-randomized, and before-and-after studies in which the intervention was delivered within community settings and the reported outcomes were relevant to the diseases under review. A separate search strategy was developed for each disease using appropriate keywords, medical subject headings $(\mathrm{MeSH})$, and free text terms. Searches were conducted in the PubMed, Cochrane Libraries, Embase, and the World Health Organization (WHO) Regional Databases. Studies that met the inclusion criteria were selected and double data was abstracted on a standardized abstraction sheet. Quality assessment of the included randomized controlled trials (RCTs) was done using the Cochrane risk of bias assessment tool [8]. The outcomes of interest assessed for each of the above diseases are outlined in Table 1. We conducted a metaanalysis for individual studies using the software Review Manager 5.1. Pooled statistics were reported as the relative risk (RR) for categorical variables and standard mean difference (SMD) for continuous variables between the experimental and control groups with 95\% confidence
Table 1 Outcomes analyzed

\begin{tabular}{|c|c|}
\hline Outcomes & Outcomes analyzed \\
\hline \multirow[t]{6}{*}{ Morbidity } & - Hookworm prevalence and intensity \\
\hline & - Ascaris prevalence and intensity \\
\hline & - Trichuris prevalence and intensity \\
\hline & - Schistosoma haematobium prevalence \\
\hline & - Schistosoma mansoni prevalence \\
\hline & - Schistosoma japonicum prevalence \\
\hline \multirow[t]{2}{*}{ Anthropometry } & - Weight \\
\hline & • Height \\
\hline \multirow[t]{3}{*}{ Hematologic } & - Prevalence of anemia \\
\hline & - Mean hemoglobin \\
\hline & - Serum ferritin \\
\hline \multirow[t]{4}{*}{ Birth outcomes } & - Birth weight \\
\hline & - Low birth weigh \\
\hline & - Very low birth weight \\
\hline & - Stillbirths \\
\hline
\end{tabular}

intervals (CIs). We also attempted to qualitatively synthesize the findings reported in the included studies for other pragmatic parameters identified in our conceptual framework including intervention coverage, challenges/barriers, enabling factors, aspects related to integrated delivery, monitoring, and evaluations and equity. The detailed methodology is described in Paper 2 of this series [7].

\section{Review}

We identified 2,556 titles from the searches conducted in all databases. After screening titles and abstracts, 208 full texts were reviewed, of which 32 studies (17 RCTs, two quasi, and 13 before-and-after studies) were included in the review (see Figure 1). The characteristics of the included studies are summarized in Table 2. Of these 32 studies, four could not be included in the metaanalysis; one study [9] did not report any outcome of interest, poolable data was not available in two studies $[10,11]$, while one [12] did not have a suitable control group for comparison. We did not find any quantifiable data from the studies on dracunculiasis, LF, and onchocerciasis to be included in the pooled analysis. For the 17 RCTs included in this review, randomization was adequate in 15 studies, allocation was concealed in six, and adequate sequence generation was also done in six. All studies provided insufficient information on selective reporting which limited us from making any judgment (see Table 3).

Included studies mainly focused on community-based MDA, which involved preventive chemotherapy in 19 of the studies and treatment after confirmed diagnosis in 11 studies. A school-based delivery strategy was the 


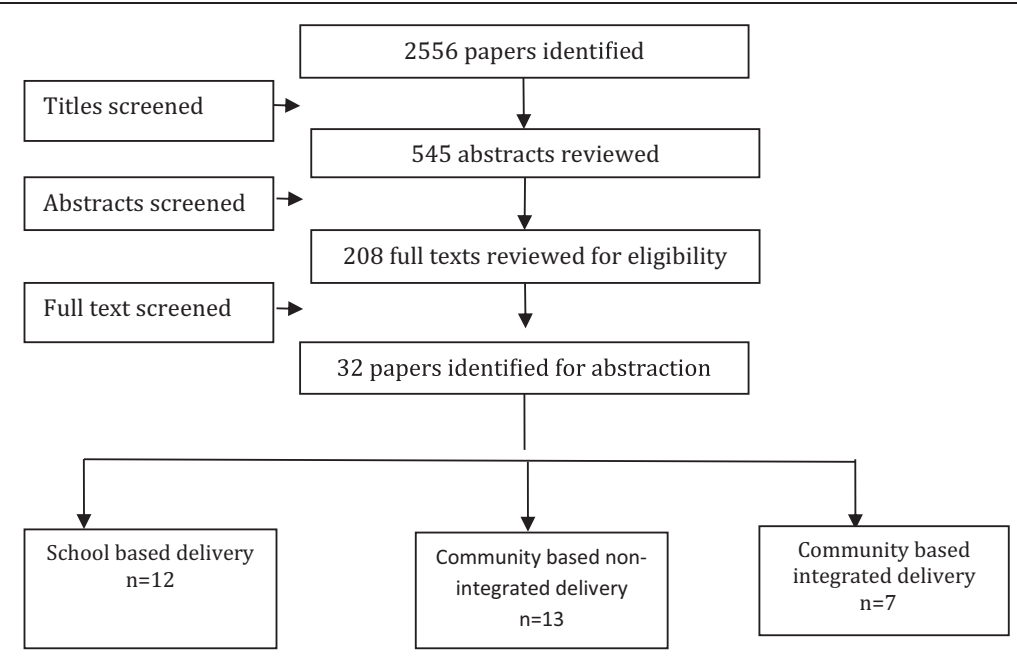

Figure 1 Search flow diagram.

most common delivery strategy used in 12 [9,13-21,24,25] studies targeting children aged five to 15 years, thirteen $[10,12,22,23,25-32,40]$ studies were non-integrated vertically delivered interventions, seven [11,33,34,36-39] were integrated with primary healthcare (PHC), and routine antenatal care (ANC) services. Almost all the studies had a component on health education to promote general hygiene and sanitation along with the drug administration. Other co-interventions included iron and $\beta$-carotene supplementation, snail control, constructing latrines, eliminating cattle from the residential areas, staff training, and community mobilization. One study [31] assessed the effectiveness of constructing a water recreation area in the community using a local lake to prevent transmission of schistosomiasis among school children. Most of the studies provided combined drug treatment for the prevention and treatment of hookworms, ascariasis, trichuriasis, and schistosomiasis with the treatment regimen including administration of albendazole (ALB) $400 \mathrm{mg}$ for $\mathrm{STH}$ and praziquantel (PZQ) $40 \mathrm{mg} / \mathrm{kg}$ for schistosomiasis, while mebendazole (MBZ) $500 \mathrm{mg}$ was used in four studies [11,14,27,37] and one study [14] also administered pyrantel-oxantel $(10 \mathrm{mg} / \mathrm{kg})$. The frequency of treatment varied according to the prevalence in the intervention area. We also attempted to conduct a subgroup analysis for the relative effectiveness of preventive and therapeutic drug administration, integrated and non-integrated delivery strategies, and evidence from RCTs/quasi and pre-post studies separately, where possible, and reported the results accordingly. The results are summarized in Tables 4 and 5.

\section{Quantitative synthesis}

Twelve studies reported STH prevalence, showing a significant 53\% reduction in the overall STH prevalence
(RR: $0.47,95 \%$ CI: $0.41,0.54)$ rate, $60 \%$ reduction in hookworm prevalence (RR: 0.40, 95\% CI: 0.31, 0.52), $68 \%$ in ascariasis (RR: $0.32,95 \%$ CI: $0.20,0.51$ ), and $34 \%$ (RR: $0.66,95 \%$ CI: $0.55,0.80$ ) in trichuriasis prevalence (see Figure 2). Twenty-five studies pooled for schistosomiasis prevalence also showed a significant $59 \%$ overall reduction (RR: $0.41,95 \%$ CI: $0.34,0.50$ ), with $59 \%$ (RR: $0.41,95 \%$ CI: $0.30,0.57$ ), 76\% (RR: $0.24,95 \% \mathrm{CI}$ : $0.07,0.80$ ) and $52 \%$ (RR: $0.48,95 \%$ CI: $0.36,0.64$ ) reductions in the prevalence of Schistosoma haematobium, Schistosoma japonicum, and Schistosoma mansoni, respectively (see Figure 3 ). CBIs also significantly reduced the mean STH intensity (SMD: $-3.16,95 \mathrm{CI}:-4.28,-2.04$ ).

Twelve studies reported on the hemoglobin $(\mathrm{Hb})$ levels and showed significantly improved mean $\mathrm{Hb}$ in the intervention group (SMD: 0.34, 95\% CI: 0.20, 0.47), while anemia significantly reduced by $10 \%$ (RR: $0.90,95 \% \mathrm{CI}$ : $0.85,0.96$ ) (see Figures 4 and 5). Impacts on serum ferritin, weight, height and delivery outcomes of stillbirth, low birth weight (LBW), and very LBW remained nonsignificant in the overall, as well as the subgroup, analyses.

Our subgroup analysis for the preventive and therapeutic drug administration showed that preventive drug administration significantly reduced the overall prevalence of STH (RR: $0.43,95 \%$ CI: 0.31, 0.59), STH intensity (SMD: $-0.22,95 \% \mathrm{CI}:-0.26,-0.17$ ), and schistosomiasis prevalence (RR: 0.39, 95\% CI: 0.27, 0.55), with nonsignificant impacts on ascariasis, trichuriasis, and Schistosoma haematobium prevalence. It also significantly improved mean $\mathrm{Hb}$ (SMD: 0.19, 95\% CI: 0.12, 0.26) and reduced anemia prevalence (RR: $0.90,95 \% \mathrm{CI}$ : $0.85,0.96$ ) and VLBW (RR: 0.38, 95\% CI: 0.16, 0.87). Preventive chemotherapy did not have a significant impact on serum ferritin, height, weight, LBW, or stillbirths. Therapeutic drug administration showed significant reductions in 
Table 2 Characteristics of the included studies

\section{Study Study design Country}

Adam 1995 [13] $\quad$ RCT $\quad$ Kenya

Ageel 1997 [33]

Pre/post

Saudi Arabia

Albonico 2002 [14]

$\mathrm{RCT}$

Tanzania

Bausch 1995 [35]

Quasi

Cameroon

Beasely 1999 [25]

$\mathrm{RCT}$

Tanzania

Bhargawa 2003 [15]

Christian 2009 [36]

Dossa 2001 [26]

$\mathrm{RC}$

$\mathrm{RCT}$

$\mathrm{RCT}$

Tanzania

Nepal

Benin

Engels 1993 [11]

Gryseels 1991 [23]

Gundersen 1990 [34]

Guyatt 2001 [16]

Halwindi 2011 [27]

Haque 2010 [28]

Hathirat 1992 [24]

Kosinski 2012 [31]

Koukounari 2006 [17]

Koukounari 2007 [18]

Larocque 2006 [37]

Ndibazza 2010 [38]
Pre/post Burundi

Pre/post

Burundi

Pre/post

Ethiopia

Pre/post

$\mathrm{RCT}$

RCT

$\mathrm{RCT}$

Pre/post

Pre/post

Pre/post

$\mathrm{RCT}$

RCT

Intervention

Three doses of $400 \mathrm{mg}$ ALB vs. identical placebo on three consecutive school days

One oral dose of PZQ $(40 \mathrm{mg} / \mathrm{kg})+$ snail/vector control + health education + community participation

MBZ $500 \mathrm{mg}$ vs. pyrantel-oxantel $(10 \mathrm{mg} / \mathrm{kg})$ vs. Children aged 6-9 years placebo

Health education + PZQ

Single dose of $400 \mathrm{mg}$ ALB + PPZQ (40 mg/kg) vs. placebo

ALB $400 \mathrm{mg}+$ PZQ single $40 \mathrm{mg} / \mathrm{kg}$ vs. placebo ALB $40 \mathrm{mg}$ in $2^{\text {nd }}+3^{\text {rd }}$ trimester

Iron (Fe) $(60 \mathrm{mg} /$ day) + ALB (200 mg for 3 days)

vs. FE + placebo vs. ALB + placebo vs. placeb

10 months FN

Single dose PZQ (40 mg/kg), 50-150 mg

levamisole or $500 \mathrm{mg} \mathrm{MBL}$ or PZQ (20 mg/kg) +

snail vector control + education

PZQ (40 mg/kg)

Vector snail control + $20 \mathrm{mg}$ oxamniquine followed by $40 \mathrm{mg}$ the next day

Single dose $400 \mathrm{mg}$ ALB + PZQ 40 mg/kg

Facility vs. community detected and treatment + training + education $+M B Z$

$400 \mathrm{mg}$ ALB + B-carotene (2 doses) vs. ALB + placebo vs. B-carotene + placebo vs. placebo + placebo

Fe 50 mg FeSo 4 daily for 2 weeks then 100 mg Children aged 9-11 years for 14 wks vs, anthelminthic

Water recreation area $+\mathrm{PZO}$

PZQ

PZQ $40 \mathrm{mg} / \mathrm{kg}+$ ALB $400 \mathrm{mg}$

MBZ $500 \mathrm{mg}$ single dose $+60 \mathrm{mg}$ FE daily vs. placebo $+\mathrm{FE}$

ALB (400 mg) vs. PZQ 40 mg/kg vs. ALB + PZQ vs. placebo

\section{Target population}

hookworms or ascariasis

General population infected with

School-based

schistosomiasis

Children aged 6-9 years School-based

Children aged $5-15$ years with

schistosomiasis

Infected children aged 7-12 years

Infected children aged 9-15 years

Pregnant women

Children aged $3-5$ years

General population

General population

General population

School children aged 8-14 years

Children aged 12-months (preventive)

Infected children aged 24-60 months

Children

Children aged 7-14 years

Children aged 6-14 years

Pregant women aged 18-44 years in the $2^{\text {nd }}$ trimester

Pregnant women

Non-integrated

$\mathrm{PHC}$

Non-integrated

$\mathrm{PHC}$

School-based

Non-integrated

Non-integrated

School-based

Non-integrated

School-based

School-based

Integrated with routine ANC

Integrated with routine ANC

Integrated with routine ANC 
Table 2 Characteristics of the included studies (Continued)

\begin{tabular}{|c|c|c|c|c|c|}
\hline Nsowah-Nuamah 2001 [12] & Pre/post & Ghana & $\begin{array}{l}\text { PZQ } 40 \mathrm{mg} / \mathrm{kg}+\text { passive edu vs. PZQ + no } \\
\text { education vs. PZQ + active edu vs. community } \\
\text { mobilization }\end{array}$ & Those aged 5 years and above & Non-integrated \\
\hline Olds 1999 [29] & $\mathrm{RCT}$ & China and Philippines & $\begin{array}{l}\text { ALB + PZQ vs. PZQ + ALB placebo vs. ALB + PZQ } \\
\text { placebo vs. placebo + placebo }\end{array}$ & Infected school aged children (4-18 years) & Non-integrated \\
\hline Palupi 1997 [30] & $\mathrm{RCT}$ & Indonesia & $\begin{array}{l}\mathrm{FE}+\mathrm{ALB} 400 \mathrm{mg} \text { once a week vs. FE once a } \\
\text { week + placebo vs. placebo alone }\end{array}$ & Children aged $2-5$ years & Non-integrated \\
\hline Phuc 2009 [40] & Pre/post & Vietnam & $\begin{array}{l}\text { IEC materials + staff training + Ferrous Sulphate/ } \\
\text { folic acid (200 mg) + ALB ( } 400 \text { mg) }\end{array}$ & Women of reproductive age ( $16-45$ years) & Non-integrated \\
\hline Rohner 2010 [19] & RCT & Cote d'Ivoire & $\begin{array}{l}\text { Iron fortified biscuits ( } 20 \mathrm{mg} \text { fe/day) + IPT-SP } \\
(500 \mathrm{mg} \text { sulphadoxine }+25 \mathrm{mg} \text { pyrimethamine) + } \\
\text { anthelminthic ( } 400 \mathrm{mg} \text {-single) + PZQ (single - } \\
40 \mathrm{mg} / \mathrm{kg} \text { ) in different combinations }\end{array}$ & School children aged 6-14 years & School-based \\
\hline Savioli 1989 [9] & Pre/post & Tanzania & PZQ & Children & School-based \\
\hline Sungthong 2002 [20] & RCT & Thailand & $\begin{array}{l}\text { Deworming ( } 400 \text { mg ALB single) + daily or } \\
\text { weekly iron }\end{array}$ & Children aged $6-13$ years & School-based \\
\hline Sinoun 2007 [10] & Pre/post & Cambodia & Universal chemotherapy with PZQ (40 mg/kg) & General population & Non-integrated \\
\hline Taylor 2001 [21] & $\mathrm{RCT}$ & South Africa & $\begin{array}{l}\text { ALB (400 mg) + PZQ ( } 40 \text { mg/kg) + FE fumigate } \\
200 \text { mg weekly vs. ALB }(400 \mathrm{mg})+\text { PZQ ( } 40 \text { mg/ } \\
\mathrm{kg})+ \text { placebo weekly vs. ALB }(400 \mathrm{mg}) \text { daily + } \\
\text { PZQ ( } 40 \mathrm{mg} / \mathrm{kg})+ \text { FE } 200 \mathrm{mg} \text { weekly vs. ALB } \\
(400 \mathrm{mg})+\text { PZQ }(40 \mathrm{mg} / \mathrm{kg})+\text { placebo weekly vs. } \\
\text { placebos + FE } 200 \mathrm{mg} \text { weekly vs. placebos }\end{array}$ & Children aged $6-15$ years & School-based \\
\hline Torlesse 2001 [39] & $\mathrm{RCT}$ & Sierra Leone & $\mathrm{FE}+\mathrm{ALB}$ vs. FE alone vs. ALB alone vs. placebo & Pregnant women (10-14 weeks) & Integrated with routine ANC \\
\hline Wang 2009 [32] & Quasi & China & $\begin{array}{l}\text { Health education + vector control + removing } \\
\text { cattle + latrines + PZQ }\end{array}$ & General population & Non-integrated \\
\hline Zhang 2007 [22] & Pre/post & Uganda & PZQ (40 mg/kg) + ALB(400 mg) + education & School children and adults & Non-integrated \\
\hline
\end{tabular}


Table 3 Quality assessment of the included RCTs

\begin{tabular}{|c|c|c|c|c|c|c|}
\hline Study & Randomization & Sequence generation & $\begin{array}{l}\text { Allocation } \\
\text { concealment }\end{array}$ & $\begin{array}{l}\text { Blinding of } \\
\text { participants }\end{array}$ & $\begin{array}{l}\text { Blinding of } \\
\text { assessors }\end{array}$ & $\begin{array}{l}\text { Selective } \\
\text { reporting }\end{array}$ \\
\hline Larocque 2006 [37] & Done & Done & Done & Done & Done & No \\
\hline Ndibazza 2010 [38] & Done & Done & Done & Done & Done & No \\
\hline Torlesse 2001 [39] & Done & Done & Not clear & Not clear & Not clear & Yes \\
\hline Haque 2010 [28] & Done & Not clear & Not clear & Done & Not clear & No \\
\hline Albonico 2002 [14] & Done & Done & Done & Done & Done & No \\
\hline Taylor 2001 [21] & Done & Not clear & Not clear & Done & Done & Not clear \\
\hline Adams 1994 [13] & Done & Not clear & Not clear & Done & Not clear & No \\
\hline Beasley 1999 [25] & Done & Not computerized but done & Not clear & Not done & Done & No \\
\hline Christian 2009 [36] & Not clear & Not clear & Not clear & Not Clear & Not Clear & Not clear \\
\hline Halwindi 2011 [27] & Not clear & Not clear & Not applicable & Not applicable & Not clear & No \\
\hline Olds 1999 [29] & Done & Not clear & Done & Done & Done & Not clear \\
\hline Rohner 2010 [19] & Done & Not clear & Done & Done & Done & No \\
\hline Bhargava 2003 [15] & Done & Not clear & Not clear & Not clear & Not clear & No \\
\hline Palupi 1997 [30] & Done & Not clear & Not clear & Not clear & Not clear & No \\
\hline Dossa 2001 [26] & Done & Not clear & Not clear & Done & Done & Yes \\
\hline Hathirat 1992 [24] & Done & Not clear & Not clear & Done & Done & No \\
\hline Sungthong 2002 [20] & Done & Done & Done & Done & Done & No \\
\hline
\end{tabular}

STH prevalence (RR: 0.52, 95\% CI: 0.44, 0.61), intensity (SMD: $-5.29,95 \%$ CI: $-9.22,-1.36$ ), and schistosomiasis prevalence (RR: $0.41,95 \%$ CI: $0.30,0.55$ ). Our analysis did not show a significant impact of therapeutic chemotherapy on serum ferritin, $\mathrm{Hb}$, weight, or height.

Findings from the subgroup analysis for school-based, non-integrated, and integrated delivery strategies suggest that school-based delivery significantly reduced STH prevalence (RR: $0.49,95 \%$ CI: $0.39,0.63$ ) and intensity (SMD: $-0.22,95 \%$ CI: $-0.26,-0.17$ ), and the prevalence of all types of schistosomiasis (RR: 0.50, 95\% CI: 0.33, 0.75), with a non-significant impact on Schistosoma mansoni. It also improved mean $\mathrm{Hb}$ (SMD: 0.24, 95\% CI: $0.16,0.32$ ) and reduced anemia prevalence (RR: 0.87, 95\% CI: 0.81-0.94) among school children. However, school-based delivery did not show any significant impact on serum ferritin, height, and weight. Nonintegrated delivery reduced STH prevalence (RR: 0.52, 95\% CI: 0.41, 0.67), intensity (SMD: $-5.29,95 \%$ CI: 9.22, -1.36), and schistosomiasis prevalence (RR: 0.42, 95\% CI: 0.31, 0.57), with a non-significant impact on Schistosoma mansoni. Integrated delivery improved schistosomiasis prevalence (RR: 0.24, 95\% CI: 0.11, 0.5) and overall STH prevalence (RR: 0.30, 95\% CI: 0.12, 0.78 ), with non-significant impacts on the prevalence of hookworm or trichuriasis.

\section{Qualitative synthesis}

Since most of the quantitative outcomes reported pertained to disease specific indicators, we also attempted to qualitatively synthesize the findings reported in the included studies on other pragmatic parameters from our conceptual framework. CBIs have been reported to achieve large-scale nationwide coverage as seen in Burkina Faso, which was the first country in the WHO African Region to achieve nationwide coverage with anthelminthic drugs against three major NTDs: LF, schistosomiasis, and STH [18]. Furthermore, when delivered in integration with the existing health systems, these programs can achieve maximum coverage as seen in the Gizan region, where a vertically-oriented program, involving mobile teams for schistosome control, failed to achieve the target of $80 \%$ population coverage and was costly to sustain. The same program, when later on integrated with the existing PHC, led to a significant increase in the numbers of patients examined and chemotherapy coverage. Similarly in Zimbabwe and Burundi, schistosomiasis and other control programs for NTDs have increasingly been integrated into horizontal PHC systems [23]. The available health infrastructure allows the control strategy to be very well integrated into basic health services, enabling it to be sustained and making it affordable for the national health budget [11]. In a health system that generally enjoys a strong structure from the provincial level down to the community level, successful integration of these services are relatively easier and just require making small modifications to the existing system. However, this process may require more attention in countries where the health service structure is weak. The PHC approach has led to better coverage as compared to the vertical programs, and 
Table 4 Results for the overall and subgroup analysis according to type of study and treatment

\begin{tabular}{|c|c|c|c|c|c|}
\hline \multirow[t]{2}{*}{ Outcomes } & \multicolumn{4}{|l|}{ Estimates $(95 \% \mathrm{Cl})$} & \multirow[b]{2}{*}{ Therapeutic } \\
\hline & Combined & RCTs & Pre-post studies & Preventive & \\
\hline \multirow[t]{2}{*}{ STH Prevalence (RR) } & $0.47[0.41,0.54]$ & $0.47[0.41,0.55]$ & $0.51[0.40,0.65]$ & $0.43[0.31,0.59]$ & $0.52[0.44,0.61]$ \\
\hline & 45 datasets from 12 studies & 40 datasets from 9 studies & 5 datasets from 3 studies & 22 datasets from 7 studies & 23 datasets from 5 studies \\
\hline \multirow[t]{2}{*}{ Hookworm } & $0.40[0.31,0.52]$ & $0.41[0.32,0.54]$ & $0.39[0.23,0.65]$ & $0.26[0.14,0.45]$ & $0.60[0.46,0.78]$ \\
\hline & 15 datasets, 10 studies & 13 datasets from 8 studies & 2 datasets from 2 studies & 8 datasets from 6 studies & 7 datasets from 4 studies \\
\hline \multirow[t]{2}{*}{ Ascariasis } & $0.32[0.20,0.51]$ & $0.30[0.18,0.49]$ & $0.61[0.41,0.90]$ & $0.62[0.38,1.01]$ & $0.18[0.08,0.40]$ \\
\hline & 15 datasets, 9 studies & 14 datasets from 8 studies & 1 dataset from 1 study & 7 datasets from 4 studies & 8 datasets from 5 studies \\
\hline \multirow[t]{2}{*}{ Trichuriasis } & $0.66[0.55,0.80]$ & $0.65[0.53,0.79]$ & $0.97[0.68,1.37]$ & $0.57[0.22,1.46]$ & $0.76[0.66,0.88]$ \\
\hline & 14 datasets, 8 studies & 13 datasets from 7 studies & 1 dataset from 1 study & 6 datasets from 3 studies & 8 datasets from 5 studies \\
\hline \multirow[t]{2}{*}{ Schistosomiasis Prevalence (RR) } & $0.41[0.34,0.50]$ & $0.42[0.33,0.54]$ & $0.37[0.25,0.55]$ & $0.39[0.27,0.55]$ & $0.41[0.30,0.55]$ \\
\hline & 25 datasets, 13 studies & 16 datasets from 6 studies & 9 datasets from 7 studies & 11 datasets from 7studies & 14 datasets from 6 studies \\
\hline \multirow[t]{2}{*}{ Schistosoma Haematobium } & $0.41[0.30,0.57]$ & $0.43[0.29,0.64]$ & $0.33[0.17,0.65]$ & $0.59[0.35,1.02]$ & $0.31[0.18,0.52]$ \\
\hline & 10 datasets, 8 studies & 6 datasets from 4 studies & 4 datasets from 4 studies & 3 datasets from 3 studies & 7 datasets from 5 studies \\
\hline \multirow[t]{2}{*}{ Schistosoma Japonicum } & $0.24[0.07,0.80]$ & $0.24[0.07,0.80]$ & No studies & $0.11[0.05,0.26]$ & $0.30[0.07,1.31]$ \\
\hline & 4 datasets, 2 studies & 4 datasets from 2 studies & & 1 dataset from 1 study & 3 datasets from 1 study \\
\hline \multirow[t]{2}{*}{ Schistosoma Mansoni } & $0.48[0.36,0.64]$ & $0.55[0.38,0.81]$ & $0.39[0.25,0.62]$ & $0.37[0.24,0.58]$ & $0.67[0.45,1.01]$ \\
\hline & 11 datasets, 7 studies & 6 datasets from 2 studies & 5 datasets from 5 studies & 7 datasets from 5 studies & 4 datasets from 2 studies \\
\hline \multirow[t]{2}{*}{ STH intensity (SMD) } & $-3.16[-4.28,-2.04]$ & $-5.29[-9.22,-1.36]$ & $-0.22[-0.26,-0.17]$ & $-0.22[-0.26,-0.17]$ & $-5.29[-9.22,-1.36]$ \\
\hline & 5 datasets, 3 studies & 3 datasets from 1 study & 2 datasets from 2 studies & 2 datasets from 2 studies & 3 datasets from 1 study \\
\hline \multirow[t]{2}{*}{ Hookworm } & $-2.22[-3.27,-1.17]$ & $-6.79[-7.44,-6.14]$ & $-0.22[-0.26,-0.17]$ & $-0.22[-0.26,-0.17]$ & $-6.79[-7.44,-6.14]$ \\
\hline & 3 datasets, 03 studies & 1 dataset from 1 study & 2 datasets from 2 studies & 2 datasets from 2 studies & 1 dataset from 1 study \\
\hline \multirow[t]{2}{*}{ Ascariasis } & $-7.25[-7.93,-6.56]$ & $-7.25[-7.93,-6.56]$ & No studies & No studies & $-7.25[-7.93,-6.56]$ \\
\hline & 1 dataset, 1 study & 1 dataset from 1 study & & & 1 dataset from 1 study \\
\hline \multirow[t]{2}{*}{ Trichuriasis } & $-1.87[-2.16,-1.57]$ & $-1.87[-2.16,-1.57]$ & No Studies & No studies & $-1.87[-2.16,-1.57]$ \\
\hline & 1 dataset, 01 study & 1 dataset from 1 study & & & 1 dataset from 1 study \\
\hline \multirow[t]{2}{*}{ Mean hemoglobin (SMD) } & $0.34[0.20,0.47]$ & $0.43[0.15,0.71]$ & $0.22[0.13,0.31]$ & $0.19[0.12,0.26]$ & $1.28[-0.53,3.08]$ \\
\hline & 14 datasets, 12 studies & 9 datasets from 7 studies & 5 datasets from 5 studies & 12 datasets from 10 studies & 2 datasets from 2 studies \\
\hline \multirow[t]{2}{*}{ Anemia } & $0.90[0.85,0.96]$ & $0.92[0.85,1.00]$ & $0.87[0.78,0.97]$ & $0.90[0.85,0.96]$ & $0.85[0.69,1.06]$ \\
\hline & 9 datasets, 7 studies & 5 datasets from 3 studies & 4 datasets from 4 studies & 8 datasets from 6 studies & 1 dataset from 1 study \\
\hline \multirow[t]{2}{*}{ Serum ferritin (SMD) } & $2.30[-1.13,5.73]$ & $0.06[-0.07,0.18]$ & $0.10[-0.16,0.37]$ & $0.09[-0.12,0.29]$ & $0.07[-0.07,0.20]$ \\
\hline & 4 datasets, 4 studies & 2 dataset from 2 studies & 2 datasets from 2 studies & 3 datasets from 3 studies & 1 dataset from 1 study \\
\hline \multirow[t]{2}{*}{ Height } & $0.01[-0.10,0.12]$ & $-0.04[-0.16,0.08]$ & $0.23[-0.02,0.49]$ & $0.08[-0.12,0.28]$ & $-0.02[-0.15,0.11]$ \\
\hline & 4 datasets, 4 studies & 3 datasets from 3 studies & 1 dataset from 1 study & 2 datasets from 2 studies & 2 datasets from 2 studies \\
\hline
\end{tabular}


Table 4 Results for the overall and subgroup analysis according to type of study and treatment (Continued)

\begin{tabular}{|c|c|c|c|c|c|}
\hline \multirow[t]{2}{*}{ Weight } & $-0.13[-0.42,0.16]$ & $-0.28[-0.66,0.11]$ & $0.22[-0.03,0.47]$ & $-0.01[-0.48,0.46]$ & $-0.35[-1.13,0.42]$ \\
\hline & 4 datasets, 4 studies & 3 datasets from 3 studies & 1 dataset from 1 study & 2 datasets from 2 studies & 2 data sets from 2 studies \\
\hline \multicolumn{6}{|c|}{ Birth outcomes } \\
\hline \multirow[t]{2}{*}{ Birth weight } & $-9.52[-13.86,-5.19]$ & $-9.52[-13.86,-5.19]$ & No studies & $-9.52[-13.86,-5.19]$ & No study \\
\hline & 6 datasets, 3 studies & 6 datasets from 3 studies & & 6 datasets from 3 studies & \\
\hline \multirow[t]{2}{*}{ LBW } & $0.96[0.78,1.18]$ & $0.96[0.78,1.18]$ & No studies & $0.96[0.78,1.18]$ & No studies \\
\hline & 4 datasets, 2 studies & 4 datasets from 2 studies & & 4 datasets from 2 studies & \\
\hline \multirow[t]{2}{*}{ VLBW } & $0.48[0.19,1.19]$ & $0.48[0.19,1.19]$ & No studies & $0.48[0.19,1.19]$ & No studies \\
\hline & 4 datasets, 2 studies & 4 datasets, 2 studies & & 4 datasets, 2 studies & \\
\hline \multirow[t]{2}{*}{ Stillbirths } & $1.54[0.93,2.58]$ & $1.54[0.93,2.58]$ & No studies & $1.54[0.93,2.58]$ & No studies \\
\hline & 3 datasets, 1 study & 3 datasets from 1 study & & 3 datasets from 1 study & \\
\hline
\end{tabular}


Table 5 Summary estimates for the overall and subgroup analysis for school-based, non-integrated, and integrated delivery strategies

\begin{tabular}{|c|c|c|c|c|}
\hline \multirow[t]{2}{*}{ Outcomes } & \multicolumn{4}{|l|}{ Estimates $(95 \% \mathrm{Cl})$} \\
\hline & Combined & School-based delivery & Community-based non-integrated delivery & Community-based integrated delivery \\
\hline \multirow[t]{2}{*}{ STH Prevalence (RR) } & $0.45[0.38,0.54]$ & $0.49[0.39,0.63]$ & $0.52[0.41,0.67]$ & $0.30[0.12,0.78]$ \\
\hline & 45 datasets, 12 studies & 19 datasets from 6 studies & 16 datasets from 4 studies & 9 datasets from 1 study \\
\hline \multirow[t]{2}{*}{ Hookworm } & $0.38[0.27,0.53]$ & $0.37[0.22,0.62]$ & $0.49[0.29,0.85]$ & $0.25[0.04,1.62]$ \\
\hline & 15 datasets, 10 studies & 7 datasets from 5 studies & 5 datasets from 3 studies & 3 datasets from 1 study \\
\hline \multirow[t]{2}{*}{ Ascariasis } & $0.32[0.19,0.52]$ & $0.28[0.09,0.89]$ & $0.36[0.18,0.74]$ & $0.28[0.09,0.87]$ \\
\hline & 15 datasets, 9 studies & 4 datasets from 6 studies & 6 datasets from 4 studies & 3 datasets from 1 studies \\
\hline \multirow[t]{2}{*}{ Trichuriasis } & $0.66[0.53,0.82]$ & $0.78[0.67,0.90]$ & $0.76[0.62,0.94]$ & $0.41[0.07,2.56]$ \\
\hline & 14 datasets, 8 studies & 4 datasets from 6 studies & 5 datasets from 3 study & 3 datasets from 1 studies \\
\hline \multirow[t]{2}{*}{ Schistosomiasis Prevalence (RR) } & $0.40[0.33,0.50]$ & $0.50[0.33,0.75]$ & $0.42[0.31,0.57]$ & $0.24[0.11,0.56]$ \\
\hline & 25 datasets, 13 studies & 7 datasets from 5 studies & 12 datasets from 4 studies & 6 datasets from 4 studies \\
\hline \multirow[t]{2}{*}{ S. Haematobium } & $0.40[0.29,0.57]$ & $0.59[0.35,1.02]$ & $0.42[0.26,0.70]$ & $0.05[0.01,0.45]$ \\
\hline & 10 datasets, 8 studies & 3 datasets from 3 studies & 5 datasets from 3 studies & 2 datasets from 2 studies \\
\hline \multirow[t]{2}{*}{ S. Japonicum } & $0.24[0.06,0.87]$ & No studies & $0.24[0.06,0.87]$ & No studies \\
\hline & 4 datasets, 2 studies & & 4 datasets from 2 studies & \\
\hline \multirow[t]{2}{*}{ S. Mansoni } & $0.48[0.35,0.65]$ & $0.44[0.27,0.74]$ & $0.68[0.42,1.10]$ & $0.38[0.16,0.91]$ \\
\hline & 11 datasets, 7 studies & 4 datasets from 4 studies & 3 datasets from 1 study & 4 datasets from 2 studies \\
\hline \multirow[t]{2}{*}{ STH intensity (SMD) } & $-3.16[-4.28,-2.04]$ & $-0.22[-0.26,-0.17]$ & $-5.29[-9.22,-1.36]$ & No studies \\
\hline & 5 datasets, 3 studies & 2 datasets, 2 studies & 3 datasets from 1 study & \\
\hline \multirow[t]{2}{*}{ Hookworm } & $-2.22[-3.27,-1.17]$ & $-0.22[-0.26,-0.17]$ & $-6.79[-7.44,-6.14]$ & No studies \\
\hline & 3 datasets, 3 studies & 2 datasets, 2 studies & 1 dataset from 1 study & \\
\hline \multirow[t]{2}{*}{ Ascariasis } & $-7.25[-7.93,-6.56]$ & No studies & $-7.25[-7.93,-6.56]$ & No studies \\
\hline & 1 dataset, 1 study & & 1 dataset from 1 study & \\
\hline \multirow[t]{2}{*}{ Trichuriasis } & $-1.87[-2.16,-1.57]$ & No studies & $-1.87[-2.16,-1.57]$ & No studies \\
\hline & 1 dataset, 1 study & & 1 dataset from 1 study & \\
\hline \multirow[t]{2}{*}{ Mean hemoglobin (SMD) } & $0.34[0.20,0.47]$ & $0.24[0.16,0.32]$ & $0.93[-0.33,2.18]$ & $0.09[-0.01,0.20]$ \\
\hline & 14 datasets, 12 studies & 7 datasets from 7 studies & 3 datasets from 3 studies & 4 datasets from 2 studies \\
\hline \multirow[t]{2}{*}{ Anemia } & $0.90[0.85,0.96]$ & $0.87[0.81,0.94]$ & $0.85[0.69,1.06]$ & $0.99[0.90,1.09]$ \\
\hline & 9 datasets, 7 studies & 5 datasets from 5 studies & 1 datasets from 1 study & 3 datasets from 1 study \\
\hline \multirow[t]{2}{*}{ Serum ferritin (SMD) } & $2.30[-1.13,5.73]$ & $0.10[-0.04,0.25]$ & No studies & $-0.01[-0.36,0.34]$ \\
\hline & 4 datasets, 4 studies & 3 datasets from 3 studies & & 1 dataset from 1 study \\
\hline Height & $0.01[-0.10,0.12]$ & $0.04[-0.08,0.15]$ & $-0.19[-0.53,0.14]$ & No studies \\
\hline
\end{tabular}


Table 5 Summary estimates for the overall and subgroup analysis for school-based, non-integrated, and integrated delivery strategies (Continued)

\section{Weight} $-0.13[-0.42,0.16]$

4 datasets, 4 studies

$-9.52[-13.86,-5.19]$

6 datasets, 3 studies

Birth weight

LBW

VLBW

Stillbirths

${ }^{*}$ Estimates in bold are statistically significant
$0.96[0.78,1.18]$
No studies

4 datasets, 2 studies

$0.38[0.16,0.87]$

4 datasets, 2 studies

$1.54[0.93,2.58]$

No studies

3 datasets, 1 study

No studies

No studies $-0.09[-0.46,0.27]$

3 datasets from 3 studies

$-0.27[-0.60,0.07]$

1 dataset from 1 study

No studies

No studies
No studies

$-9.52[-13.86,-5.19]$

6 datasets from 3 studies

$0.96[0.78,1.18]$

4 datasets from 2 studies

$0.38[0.16,0.87]$

4 datasets from 2 studies

$1.54[0.93,2.58]$

3 datasets from 1 study 


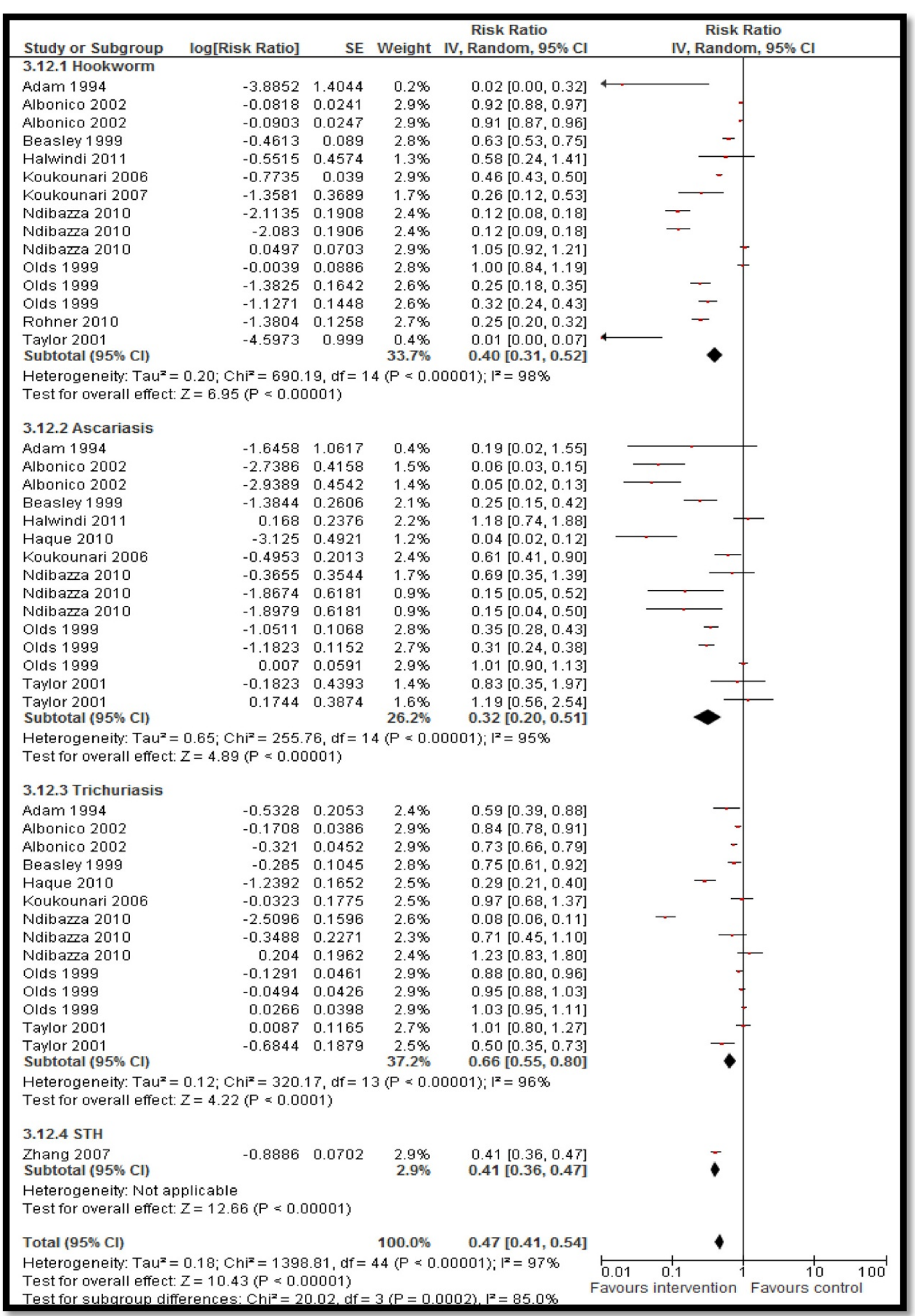

Figure 2 Forest plot for the impact of CBIs on STH prevalence. 


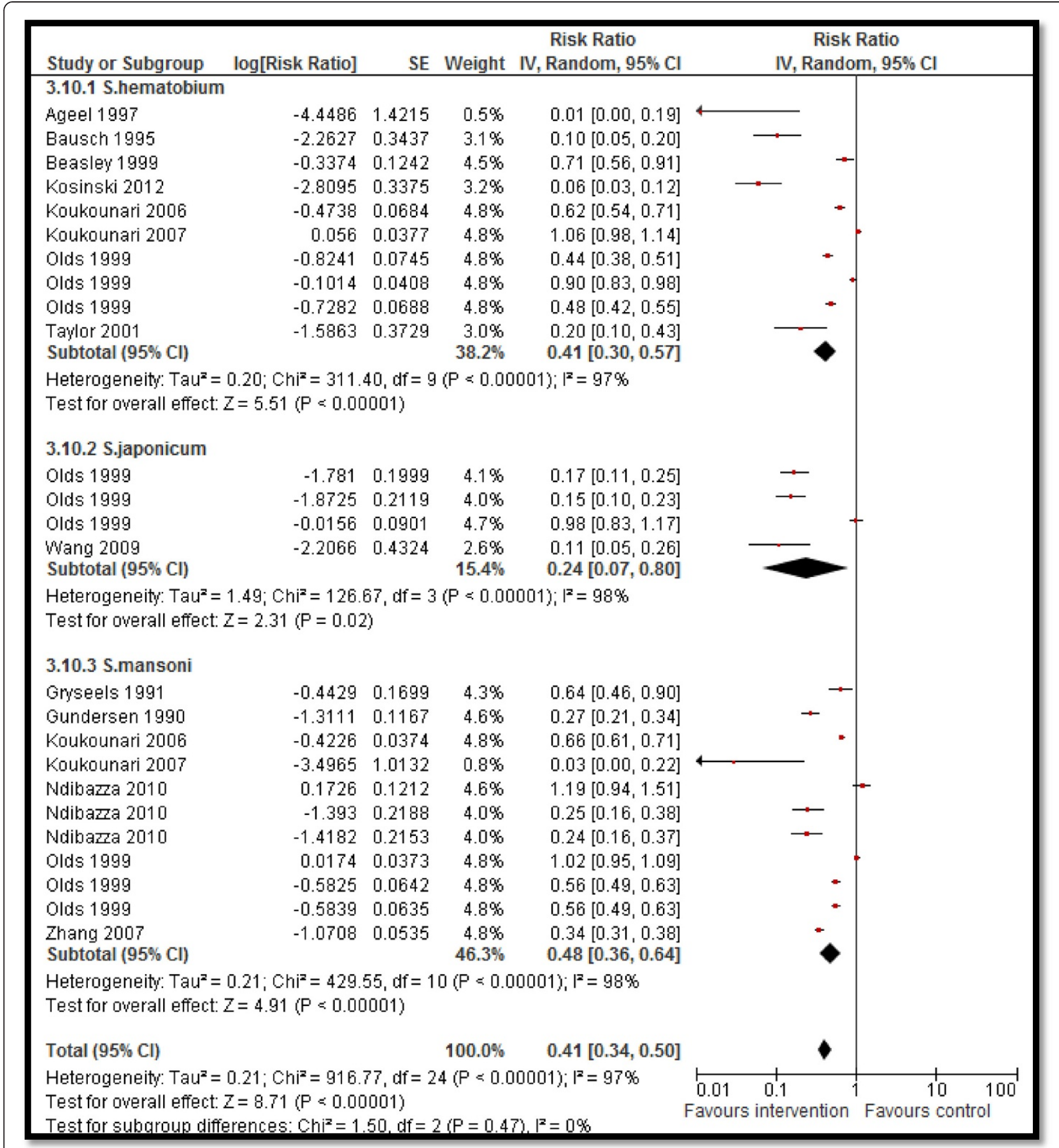

Figure 3 Forest plot for the impact of CBIs on schistosomiasis.

has been effective in reducing the overall prevalence of helminthic infections.

CBIs have achieved a higher coverage without any increase in implementation costs at the district and health facility levels. At the community level, there is an increase in 'opportunity costs' from community implementers who volunteer their time, thus forgoing other remunerative activities. Some of the studies reported no major direct additional costs incurred because the drugs, training materials, and stationary were provided by the PHC from their available resources, and the community health workers (CHWs) were not given any monetary incentives. Hence, the major additional costs included the indirect cost of labour hours for the CHWs and the PHC staff during training and implementation of the program. However, for national scale-up, the biggest 


\begin{tabular}{|c|c|c|c|c|c|c|c|c|c|c|}
\hline \multirow[b]{2}{*}{ Study or Subgroup } & \multicolumn{3}{|c|}{ Experimental } & \multicolumn{3}{|c|}{ Control } & \multicolumn{2}{|r|}{ Std. Mean Difference } & \multirow{2}{*}{\multicolumn{2}{|c|}{$\begin{array}{l}\text { Std. Mean Difference } \\
\text { IV, Random, } 95 \% \mathrm{Cl}\end{array}$}} \\
\hline & Mean & SD & Total & Mean & SD & Total & Weight & IV, Random, $95 \% \mathrm{Cl}$ & & \\
\hline Beasley 1999 & 10.9 & 0.08 & 127 & 10.7 & 0.1 & 123 & $5.7 \%$ & $2.21[1.89,2.52]$ & & b \\
\hline Bhargawa 2003 & 121 & 12.5 & 331 & 116.1 & 14 & 602 & $7.7 \%$ & $0.36[0.23,0.50]$ & & $\rightarrow$ \\
\hline Dossa 2001 & 109.3 & 11.5 & 72 & 108.6 & 11 & 68 & $5.6 \%$ & $0.06[-0.27,0.39]$ & & \\
\hline Guyatt 2001 & 112.8 & 15.1 & 1121 & 107.3 & 14.5 & 466 & $7.9 \%$ & $0.37[0.26,0.48]$ & & - \\
\hline Hathirat 1992 & 124 & 9.7 & 887 & 123 & 11.3 & 888 & $8.0 \%$ & $0.09[0.00,0.19]$ & & - \\
\hline Koukounari 2006 & 11.67 & 1.45 & 2682 & 11.43 & 1.45 & 2682 & $8.3 \%$ & $0.17[0.11,0.22]$ & & - \\
\hline Koukounari 2007 & 11.25 & 1.2 & 1131 & 10.97 & 1.46 & 1131 & $8.1 \%$ & $0.21[0.13,0.29]$ & & - \\
\hline Ndibaza 2010 (1) & 11.84 & 1.96 & 500 & 11.69 & 1.98 & 472 & $7.8 \%$ & $0.08[-0.05,0.20]$ & & $f-$ \\
\hline Ndibaza 2010 (2) & 11.89 & 1.87 & 458 & 11.69 & 1.98 & 472 & $7.8 \%$ & $0.10[-0.02,0.23]$ & & $=$ \\
\hline Ndibaza 2010 (3) & 11.73 & 1.93 & 488 & 11.69 & 1.98 & 472 & $7.8 \%$ & $0.02[-0.11,0.15]$ & & - \\
\hline Palupi 1997 & 120.1 & 9.7 & 95 & 115.2 & 9.4 & 98 & $6.1 \%$ & $0.51[0.22,0.80]$ & & \\
\hline Rohner 2010 & 109.4 & 9.5 & 290 & 107.4 & 9.4 & 301 & $7.5 \%$ & $0.21[0.05,0.37]$ & & $\rightarrow$ \\
\hline Sungthong 2002 & 125.3 & 9.3 & 121 & 121.8 & 9.8 & 121 & $6.5 \%$ & $0.37[0.11,0.62]$ & & $\rightarrow$ \\
\hline Torlesse 2001 & 98.6 & 13.3 & 61 & 92.8 & 12 & 64 & $5.3 \%$ & $0.46[0.10,0.81]$ & & \\
\hline Total $(95 \% \mathrm{Cl})$ & & & 8364 & & & 7960 & $100.0 \%$ & $0.34[0.20,0.47]$ & & \\
\hline $\begin{array}{l}\text { Heterogeneity: Tauz = } \\
\text { Test for overall effect: } \\
\text { (1) ALB+PZQ } \\
\text { (2) PZQ alone } \\
\text { (3) ALB alone }\end{array}$ & $\begin{array}{l}0.06 ; \mathrm{Cl} \\
Z=4.87\end{array}$ & $\begin{array}{l}h i^{2}=10 \\
(P=0\end{array}$ & $\begin{array}{l}99.11,0 \\
.00001\end{array}$ & $\begin{array}{l}d f=13( \\
1)\end{array}$ & $P=0.1$ & 00001) & $I^{2}=93 \%$ & & $\begin{array}{cc}1 & 1 \\
-1 & -0.5 \\
\text { Favours control }\end{array}$ & $\begin{array}{ccc} & 1 & 1 \\
0 & 0.5 & 1 \\
\text { Favours intervention }\end{array}$ \\
\hline
\end{tabular}

Figure 4 Forest plot for the impact of CBIs on hemoglobin.

challenge is likely to be the cost of training and incentives for the CHWs [27]. Similarly, school-based delivery of preventive and promotive interventions is also reported to be cost effective, however, its success depended on close supervision of the teachers' compliance with the program implementation, collaboration of the educational authorities, and enthusiastic participation of the school personnel [24]. On the contrary, in some endemic areas, an integrated delivery strategy has not been successful in improving coverage because of the poor access to health services in remote areas. In view of the strong commitment of national health authorities and funding agencies to increase PHC coverage, rapid improvement of this situation can be expected. For sustained

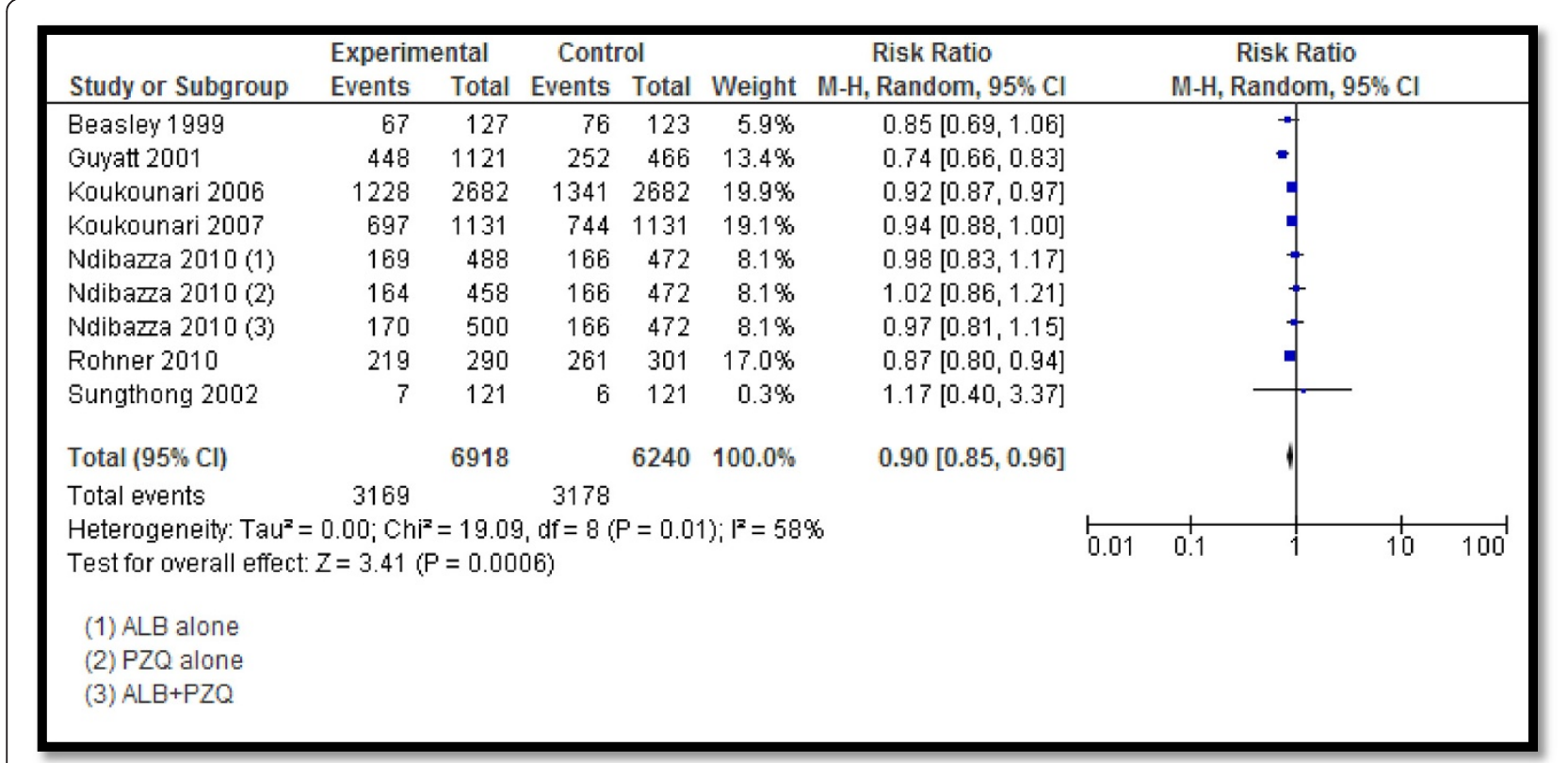

Figure 5 Forest plot for the impact of CBIs on anemia. 
reduction in infection prevalence and complete eradication, periodic implementation is also necessary to ensure continuity of morbidity control and achieve universal coverage. In endemic areas, an intermittent intervention delivery has shown to reduce the prevalence but did not completely eradicate the infections. This might be attributable to the fact that chemotherapy reduces disease prevalence only at the time of its administration and does not prevent reinfection [32]. Hence, in endemic areas, periodic implementation along with efforts to prevent reinfection is required.

Most of the studies support a multi-component strategy involving chemotherapy, health education, improved water supply and sanitation, and snail control. Health education and community involvement have been highlighted as essential components in any strategy for helminthic infection control and have been used in many countries $[12,22,33]$. Examples of such successful programs are the chemotherapy program in Sudan where village health committees served as facilitators, and in Zimbabwe where CHWs were involved in the implementation of improvements to the water supply, sanitation, and in health education programs. Similarly in Ethiopia and Egypt, local health personnel, farmers, school health visitors, and teachers assisted in the implementation of successful control programs. Knowledge and education plays an important role as, apparently, the information motivates the participants and consequently ensures a higher level of compliance [30,40]. The process of designing the program, from its very initiation, should include open discussions and involvement of all partners from the international to the local level. This will ensure that everyone understands the goals, benefits and underlying principles of the project, and have the flexibility needed to adapt to particular local conditions in order to achieve these goals [40]. Strong commitment of all partners to the intervention has reportedly led to excellent participation levels and a significant clinical impact [34,40]. Besides health education and community participation, the socioeconomic development leading to a general rise in living standards, improved sanitation and water supplies, construction of drilled wells in rural areas, and a significant increase in medical care also played a large part in the control of helminthic NTDs [33]. Another important enabling factor was free distribution of drugs and supplies to ensure equitable distribution to poor and marginalized groups, which also helped avoid a complicated system of cost recovery and achieve high compliance levels.

Financial, logistical, and organizational limitations were the major reported constraints in the long-term sustainability of such programs. Despite considerable efforts and their high costs, the results of some of the vector control programs have been disappointing, largely because of poor weed control, lack of maintenance, and the operational difficulty of covering entire networks, which leads to rapid recolonization of treated sites. Large-scale health education programs will not serve their purpose if alternatives to traditional water contact activities are not available. Maintenance is still reported as a major hindrance and local authorities should try to find adequate solutions. Annual tax payments, private latrine programs, subsidized prices, and credit facilities are some of the counter strategies devised to overcome these issues and ensure sustainability of such programs after withdrawal of external funds [11]. Another major challenge is to maintain a high level of participation and enthusiasm as the project is integrated into the routine provincial health program [40]. Although maximum coverage has been achieved in the school-based programs, frequent migration of people (particularly from the fishing communities), influx from neighboring countries, and absence from school of some school-aged children remain to be significant hurdles to the successful implementation of the program [23]. Although often disregarded, religious, economic, and educational heterogeneity within communities may also impair disease control efforts and, hence, these subtle demographic variables must be taken into account.

\section{Discussion}

The evidence in this review comes from efficacy and effectiveness studies on CBIs for helminthic NTDs compared to routine facility-based care or no intervention. Our review findings suggest that CBIs are effective in reducing the prevalence of STH and schistosomiasis, and STH intensity. They are also effective in improving mean $\mathrm{Hb}$ and reducing anemia prevalence, but there was non-significant impact on serum ferritin, height and weight gain, LBW, and stillbirths. School-based delivery was identified as a potential medium of delivery as it significantly reduced STH and schistosomiasis prevalence, STH intensity, and anemia prevalence, however, there was limited data available on the effectiveness of the integrated and non-integrated delivery strategies. The qualitative synthesis from the included studies supports community-based delivery strategies and suggests that integrated infection prevention and control measures are more effective in achieving greater coverage compared to the routine vertical delivery, albeit it requires an existing strong healthcare infrastructure. Systematic reviews have been conducted on helminthic NTDs previously, however, their scopes were either limited to a particular infection, drug efficacy or intervention, or the review did not evaluate the effectiveness of community delivery strategies [41-45].

The WHO recommends periodic administration of anthelminthic medicines, mainly ALB and MBZ for STH, 
PZQ for schistosomiasis, and ivermectin or diethylcarbamazine citrate (DEC) for LF once or twice a year depending on the baseline prevalence of the infection to control morbidity among the population at risk [46].

Health education is an essential component in the prevention and control of helminthiasis. Almost all the studies in this review had a health education component focusing on general hygiene and sanitation along with the recommended drug administration. We did not find any quantifiable data from studies on dracunculiasis, LF, and onchocerciasis to be included in the pooled analysis. Among these diseases, much progress has been made for dracunculiasis and a significant reduction in dracunculiasis prevalence of more than $99 \%$ has been achieved since 1989 [47]. Dracunculiasis is successfully at the verge of eradication due to a combination of interventions including community-based surveillance systems, intensified case-containment measures, and access to safe drinking water. Globally, 1.39 billion people still require preventive chemotherapy for LF while 123 million people are at risk of becoming infected with onchocerciasis $[46,48]$. Programs targeting LF and onchocerciasis are in place in endemic countries, for example, the Global Programme to Eliminate LF and the African Programme for Onchocerciasis. These programs enable resourcelimited countries to make medicines freely available and allow easy access to healthcare with a consequent reduction in infection prevalence [49].

Community delivery platforms are increasingly being advocated for the prevention and control of major health issues such as nutrition and childhood infection, and hence could be feasible for helminthic NTDs [50,51]. Under these community platforms, integration of various disease specific programs is being encouraged for onchocerciasis, LF, schistosomiasis, STH, and trachoma $[52,53]$. The integrated delivery of interventions to control these diseases is more feasible and cost effective as these diseases are endemic in specific geographical pockets where population are mostly co-infected and control mainly involves regular MDA of effective preventive chemotherapy. Therefore, a package of drugs for more than one NTD can be feasibly delivered. Critical factors for successful integration include active support of relevant political and health authorities, a clear understanding by all parties of the health issues, and a simple distribution process. However, there is limited data available to gauge the effectiveness of integrated delivery of these programs along with other health programs including routine ANC and PHC setups [54,55]. Schoolbased delivery has proven to be effective for the management and control of helminthic diseases as it requires a minimum amount of training for school teachers to aid implementation and hence doesn't require an added workforce, whilst providing vast coverage at low costs [56].

\section{Conclusion}

Current evidence emphasizes that effective communitybased strategies exist and deliver a range of preventive, promotive, and therapeutic interventions to combat helminthic NTDs. However, there is a need to conduct high-quality studies on the process of developing and implementing an efficient integrated program as previous global programs have focused on the control of a single NTD through a comprehensive approach. These interventions exist within the current health systems in most of the low- and middle-income countries, but there is a need to implement them on a larger scale especially to reach the unreachable.

\section{Additional file}

Additional file 1: Multilingual abstracts in the six official working

languages of the United Nations.

\section{Abbreviations}

ANC: Antenatal care; CBI: Community based intervention; CHW: Community health worker; IDoP: Infectious diseases of poverty; LF: Lymphatic filariasis; MDA: Mass drug administration; NTD: Neglected tropical disease; PHC: Primary healthcare; STH: Soil-transmitted helminthiasis; WHO: World health organization.

\section{Competing interests}

The authors declare that they have no financial or non-financial competing interests.

\section{Authors' contributions}

ZAB was responsible for designing and coordinating the review. ZSL and HM were responsible for the data collection, screening of the search results, screening of the retrieved papers against the inclusion criteria, appraising the quality of papers, and abstracting the data. RAS, JKD, and ZSL were responsible for data interpretation and writing the review. ZAB critically reviewed and modified the manuscript. All authors read and approved the final manuscript.

\section{Authors' information}

Zulfigar A Bhutta: Founding Director, Center of Excellence in Women \& Child Health, The Aga Khan University, Karachi, Pakistan and Robert Harding Chair in Global Child Health \& Policy, Center for Global Child Health, Hospital for Sick Children, Toronto, Canada

\section{Acknowledgements}

The collection of scoping reviews in this special issue of Infectious Diseases of Poverty was commissioned by the UNICEF/UNDP/World Bank/WHO Special Programme for Research and Training in Tropical Diseases (TDR) in the context of a Contribution Agreement with the European Union for "Promoting research for improved community access to health interventions in Africa".

\section{Author details}

${ }^{1}$ Division of Women and Child Health, The Aga Khan University, Karachi 74800, Pakistan. ${ }^{2}$ Brown University, Providence, RI, USA. ${ }^{3}$ Center of Excellence in Women \& Child Health, The Aga Khan University, Karachi, Pakistan. ${ }^{4}$ Center for Global Child Health Hospital for Sick Children, Toronto, Canada.

Received: 5 January 2014 Accepted: 3 July 2014

Published: 31 July 2014 


\section{References}

1. Bhutta ZA, Sommerfeld J, Lassi ZS, Salam RA, Das JK: Global Burden, Distribution and Interventions for the Infectious Diseases of Poverty. Infect Dise of Pov 2014, 3:21.

2. Hotez PJ, Bundy DAP, Beegle K, Brooker S, Drake L, de Silva N, Montresor A, Engels D, Jukes M, Chitsulo L: Helminth infections: soil-transmitted helminth infections and schistosomiasis; 2006.

3. Crompton DWT, Nesheim MC: Nutritional impact of intestinal helminthiasis during the human life cycle. Annu Rev Nutr 2002, 22(1):35-59.

4. Miguel $\mathrm{E}$, Kremer $\mathrm{M}$ : Worms: identifying impacts on education and health in the presence of treatment externalities. Econometrica 2004, 72(1):159-217

5. Hotez PJ, Molyneux DH, Fenwick A, Kumaresan J, Sachs SE, Sachs JD, Savioli L: Control of neglected tropical diseases. N Engl J Med 2007, 357(10):1018-1027.

6. Hawley WA, Phillips-Howard PA, Kuile FO, Terlouw DJ, Vulule JM, Ombok M, Nahlen BL, Gimnig JE, Kariuki SK, Kolczak MS: Community-wide effects of permethrin-treated bed nets on child mortality and malaria morbidity in western Kenya. Am J Trop Med Hyg 2003, 68(4):121-127.

7. Lassi ZS, Salam RA, Das JK, Bhutta ZA: Conceptual framework and assessment methodology for the systematic review on community based interventions for the prevention and control of IDoP. Infect Dise of Pov 2014, 3:22.

8. In Cochrane Handbook for Systematic Reviews of Interventions Version 5.1.0. [updated March 2011]. Edited by H. JPT and G. S. The Cochrane Collaboration; 2011.

9. Savioli L, Dixon H, Kisumku UM, Mott KE: Control of morbidity due to Schistosoma haematobium on Pemba island; selective population chemotherapy of schoolchildren with haematuria to identify high-risk localities. Trans R Soc Trop Med Hyg 1989, 83(6):805-810.

10. Sinuon M, Tsuyuoka R, Socheat D, Odermatt P, Ohmae H, Matsuda H, Montresor A, Palmer K: Control of Schistosoma mekongi in Cambodia: results of eight years of control activities in the two endemic provinces. Trans R Soc Trop Med Hyg 2007, 101(1):34-39.

11. Engels D, Ndoricimpa J, Gryseels B: Schistosomiasis mansoni in Burundi: progress in its control since 1985. Bull World Health Organ 1993, 71(2):207-214

12. Nsowah-Nuamah NN, Mensah G, Aryeetey ME, Wagatsuma Y, Bentil G: Urinary schistosomiasis in southern Ghana: a logistic regression approach to data from a community-based integrated control program. Am J Trop Med Hyg 2001, 65(5):484-490.

13. Adams EJ, Stephenson LS, Latham MC, Kinoti SN: Physical activity and growth of Kenyan school children with hookworm, Trichuris trichiura and Ascaris lumbricoides infections are improved after treatment with albendazole. J Nutr 1994, 124(8):1199.

14. Albonico M, Bickle Q, Haji HJ, Ramsan M, Khatib KJ, Montresor A, Savioli L, Taylor M: Evaluation of the efficacy of pyrantel-oxantel for the treatment of soil-transmitted nematode infections. Trans R Soc Trop Med Hyg 2002, 96(6):685-690

15. Bhargava A, Jukes M, Lambo J, Kihamia CM, Lorri W, Nokes C, Drake L, Bundy D: Anthelmintic treatment improves the hemoglobin and serum ferritin concentrations of Tanzanian schoolchildren. Food Nutr Bull 2003, 24(4):332-342.

16. Guyatt HL, Brooker S, Kihamia CM, Hall A, Bundy DAP: Evaluation of efficacy of school-based anthelmintic treatments against anaemia in children in the United Republic of Tanzania. Bull World Health Organ 2001, 79(8):695-703.

17. Koukounari A, Fenwick A, Whawell S, Kabatereine NB, Kazibwe F, Tukahebwa EM, Stothard JR, Donnelly CA, Webster JP: Morbidity indicators of Schistosoma mansoni: relationship between infection and anemia in Ugandan schoolchildren before and after praziquantel and albendazole chemotherapy. Am J Trop Med Hyg 2006, 75(2):278-286.

18. Koukounari A, Gabrielli AF, Toura S, BosquaOliva E, Zhang Y, Sellin B, Donnelly CA, Fenwick A, Webster JP: Schistosoma haematobium infection and morbidity before and after large-scale administration of praziquantel in Burkina Faso. J Infect Dis 2007, 196(5):659.

19. Rohner F, Zimmermann MB, Amon RJ, Vounatsou P, Tschannen AB, N'Goran EK, Nindjin C, Cacou MC, TaBonla MD, Aka H: In a randomized controlled trial of iron fortification, anthelmintic treatment, and intermittent preventive treatment of malaria for anemia control in Ivorian children, only anthelmintic treatment shows modest benefit. J Nutr 2010, 140(3):635-641.

20. Sungthong R, Mo-suwan L, Chongsuvivatwong V, Geater AF: Once weekly is superior to daily iron supplementation on height gain but not on hematological improvement among schoolchildren in Thailand. J Nutr 2002, 132(3):418-422.

21. Taylor M, Jinabhai CC, Couper I, Kleinschmidt I, Jogessar VB: The effect of different anthelmintic treatment regimens combined with iron supplementation on the nutritional status of schoolchildren in KwaZuluNatal, South Africa: a randomized controlled trial. Trans R Soc Trop Med Hyg 2001, 95(2):211-216.

22. Zhang Y, Koukounari A, Kabatereine N, Fleming F, Kazibwe F, Tukahebwa E, Stothard JR, Webster J, Fenwick A: Parasitological impact of 2-year preventive chemotherapy on schistosomiasis and soil-transmitted helminthiasis in Uganda. BMC Med 2007, 5(1):27.

23. Gryseels B, Nkulikyinka L, Engels D: Repeated community-based chemotherapy for the control of Schistosoma mansoni: effect of screening and selective treatment on prevalences and intensities of infection. Am J Trop Med Hyg 1991, 45(4):509-517.

24. Hathirat P, Valyasevi A, Kotchabhakdi NJ, Rojroongwasinkul N, Pollitt E: Effects of an iron supplementation trial on the Fe status of Thai schoolchildren. Br J Nutr 1992, 68(1):245-252.

25. Beasley NMR, Tomkins AM, Hall A, Kihamia CM, Lorri W, Nduma B, Issae W, Nokes C, Bundy DAP: The impact of population level deworming on the haemoglobin levels of schoolchildren in Tanga, Tanzania. Trop Med Int Health 1999, 4(11):744-750.

26. Dossa RAM, Ategbo EAD, De Koning F, Van Raaij JMA, Hautvast J: Impact of iron supplementation and deworming on growth performance in preschool Beninese children. Eur J Clin Nutr 2001, 55(4):223-228.

27. Halwindi $H$, Magnussen $P$, Siziya S, Handema RAY, Meyrowitsch D, Olsen A: Impact of community-directed treatment on soil transmitted helminth infections in children aged 12 to 59 months in Mazabuka District, Zambia. Parasitology 2011, 138(12):1578

28. Haque $\mathrm{R}$, Ahmed T, Wahed MA, Mondal D, Rahman ASMH, Albert MJ: Low-dose $\hat{I}^{2}$-carotene supplementation and deworming improve serum vitamin $A$ and $\hat{l}^{2}$-carotene concentrations in preschool children of Bangladesh. J Health Popul Nutr 2010, 28(3):230.

29. Olds GR, King C, Hewlett J, Olveda R, Wu G, Ouma J, Peters P, McGarvey S, Odhiambo O, Koech D: Double-blind placebo-controlled study of concurrent administration of albendazole and praziquantel in schoolchildren with schistosomiasis and geohelminths. J Infect Dis 1999, 179(4):996-1003.

30. Palupi L, Schultink W, Achadi E, Gross R: Effective community intervention to improve hemoglobin status in preschoolers receiving once-weekly iron supplementation. Am J Clin Nutr 1996, 65(4):1057-1061.

31. Kosinski KC, Adjei MN, Bosompem KM, Crocker JJ, Durant JL, Osabutey D, Plummer JD, Stadecker MJ, Wagner AD, Woodin M, Gute D: Effective control of Schistosoma haematobium infection in a Ghanaian community following installation of a water recreation area. PLoS Negl Trop Dis 2012, 6(7):e1709.

32. Wang LD, Chen HG, Guo JG, Zeng XJ, Hong XL, Xiong JJ, Wu XH, Wang XH, Wang LY, Xia G, Hao Y, Chin D, Zhou XN: A strategy to control transmission of Schistosoma japonicum in China. N Engl J Med 2009, 360(2):121-128.

33. Ageel ARM, Amin MA: Integration of schistosomiasis control activities into the primary health care system in the Gizan region, Saudi Arabia. Ann Trop Med Parasitol 1997, 91(8):907-916.

34. Gundersen SG, Birrie H, Torvik HP, Scherbaum H: Control of Schistosoma mansoni in the Blue Nile Valley of western Ethiopia by mass chemotherapy and focal snail control: a primary health care experience. Trans R Soc Trop Med Hyg 1990, 84(6):819-825.

35. Bausch D, Cline BL: The impact of control measures on urinary schistosomiasis in primary school children in northern Cameroon: a unique opportunity for controlled observations. Am J Trop Med Hyg 1995, 53(6):577-580

36. Christian P, Stewart CP, LeClerq SC, Wu L, Katz J, West KP, Khatry SK Antenatal and postnatal iron supplementation and childhood mortality in rural Nepal: a prospective follow-up in a randomized, controlled community trial. Am J Epidemiol 2009, 170(9):1127-1136.

37. Larocque R, Casapia M, Gotuzzo E, MacLean JD, Soto JC, Rahme E, Gyorkos TW: A double blind randomized controlled trial of antenatal 
mebendazole to reduce low birthweight in a hookworm endemic area of Peru. Trop Med Int Health 2006, 11(10):1485-1495.

38. Ndibazza J, Muhangi L, Akishule D, Kiggundu M, Ameke C, Oweka J, Kizindo R, Duong T, Kleinschmidt I, Muwanga M: Effects of deworming during pregnancy on maternal and perinatal outcomes in Entebbe, Uganda: a randomized controlled trial. Clin Infect Dis 2010, 50(4):531-540.

39. Torlesse $H$, Hodges M: Albendazole therapy and reduced decline in haemoglobin concentration during pregnancy (Sierra Leone). Trans R Soc Trop Med Hyg 2001, 95(2):195-201.

40. Phuc T, Mihrshahi S, Casey G, Phu L, Tien N, Caruana S, Thach T, Montresor A, Biggs BA: Lessons learned from implementation of a demonstration program to reduce the burden of anemia and hookworm in women in Yen Bai Province, Viet Nam. BMC Public Health 2009, 9(1):266.

41. Dickson R, Awasthi S, Williamson P, Demellweek C, Garner P: Effects of treatment for intestinal helminth infection on growth and cognitive performance in children: systematic review of randomised trials. BMJ 2000, 320:1697-1701.

42. Uneke CJ: Soil transmitted helminth infections and schistosomiasis in school age children in sub-Saharan Africa: efficacy of chemotherapeutic intervention since World Health Assembly Resolution 2001. Tanzan J Health Res 2010, 12(1):86-99.

43. Ziegelbauer K, Speich B, Mausezahl D, Bos R, Keiser J, Utzinger J: Effect of sanitation on soil-transmitted helminth infection: systematic review and meta-analysis. PLoS Med 2012, 9(1):e1001162.

44. Keiser J, Utzinger J: Efficacy of current drugs against soil-transmitted helminth infections: systematic review and meta-analysis. JAMA 2008, 299(16):1937-1948

45. Taylor-Robinson DC, Maayan N, Soares-Weiser K, Donegan S, Garner P: Deworming drugs for soil-transmitted intestinal worms in children: effects on nutritional indicators, haemoglobin and school performance. Cochrane Database Syst Rev 2012, 7(11):CD000371.

46. World Health, Organization: Sustaining the drive to overcome the global impact of neglected tropical diseases, Second WHO report on neglected tropical diseases. Geneva, Switzerland: World Health Organization; 2013.

47. World Health, Organization: Dracunculiasis eradication-global surveillance summary, 2009. Wkly Epidemiol Rec 2010, 85:165-176.

48. Hotez PJ: Forgotten people, forgotten diseases: the neglected tropical diseases and their impact on global health and development. Amer Society for Microbiology 2008.

49. World Health Organization, Dept. of Control of Neglected Tropical D, Crompton DWT, Daumerie D, Peters P, Savioli L: Working to overcome the global impact of neglected tropical diseases: first WHO report on neglected tropical diseases. Geneva, Switzerland: World Health Organization; 2010.

50. Bhutta ZA, Das JK, Rizvi A, Gaffey MF, Walker N, Horton S, Webb P, Lartey A, Black RE: Evidence-based interventions for improvement of maternal and child nutrition: what can be done and at what cost? Lancet 2013, 382(9890):452-477.

51. Bhutta ZA, Das JK, Walker N, Rizvi A, Campbell H, Rudan I, Black RE: Interventions to address deaths from childhood pneumonia and diarrhoea equitably: what works and at what cost? Lancet 2013, 381 (9875):1417-1429.

52. Brady MA, Hooper PJ, Ottesen EA: Projected benefits from integrating NTD programs in sub-Saharan Africa. Trends Parasitol 2006, 22(7):285-291

53. Hotez P, Raff S, Fenwick A, Richards F Jr, Molyneux DH: Recent progress in integrated neglected tropical disease control. Trends Parasitol 2007, 23(11):511-514.

54. Grepin KA, Reich MR: Conceptualizing integration: a framework for analysis applied to neglected tropical disease control partnerships. PLoS Negl Trop Dis 2008, 2(4):e174.
55. Lammie PJ, Fenwick A, Utzinger J: A blueprint for success: integration of neglected tropical disease control programmes. Trends Parasitol 2006, 22(7):313-321.

56. Molyneux DH: Combating the "other diseases" of MDG 6: changing the paradigm to achieve equity and poverty reduction? Trans $R$ Soc Trop Med Hyg 2008, 102(6):509-519.

doi:10.1186/2049-9957-3-23

Cite this article as: Salam et al:: Community-based interventions for the prevention and control of helmintic neglected tropical diseases. Infectious Diseases of Poverty 2014 3:23.

\section{Submit your next manuscript to BioMed Central and take full advantage of:}

- Convenient online submission

- Thorough peer review

- No space constraints or color figure charges

- Immediate publication on acceptance

- Inclusion in PubMed, CAS, Scopus and Google Scholar

- Research which is freely available for redistribution

Submit your manuscript at www.biomedcentral.com/submit
C Biomed Central 